\title{
A Pilot Study Assessing Aspects of Sexual Function Predicted to Be Important After Treatment for Prostate Cancer in Gay Men: An Underserved Domain Highlighted
}

\author{
Channa Amarasekera, MD, Vincent Wong, MD, Kathryn Jackson, MS, Emily Yura, MD, \\ Mehul Patel, MD, Adarsh Manjunath, MD, and Shilajit Kundu, MD
}

\begin{abstract}
Purpose: Existing questionnaires assessing sexual function after prostate cancer (PCa) were developed in predominantly heterosexual male cohorts and may measure function incompletely in gay men. We sought to determine if there are sexual function domains relevant to gay men that are not captured by the Expanded Prostate Cancer Index Composite (EPIC) sexual function assessment.

Methods: Fifty-three gay men with PCa responded to an online survey regarding the applicability of the sexual function domain in the validated EPIC questionnaire. They were then queried about whether the prostate is a source of sexual pleasure and the importance of measuring sexual satisfaction as it relates to receptive anal intercourse.

Results: A majority of gay men with PCa found the EPIC sexual function tool to be applicable when measuring erectile function (76.5\%). Of the men queried, $64.2 \%$ felt that the prostate is a source of sexual pleasure and $52.8 \%$ felt it important to measure sexual satisfaction associated with receptive anal intercourse. A larger proportion of gay men who engaged in receptive anal intercourse, compared with those who did not engage in receptive anal intercourse, felt that the prostate is a source of sexual pleasure (100\% vs. 57.1\%), and thought it important to measure sexual satisfaction as it relates to receptive anal intercourse after PCa treatment (90.0\% vs. $45.2 \%)$.

Conclusions: Our findings highlight the need to create a validated questionnaire to measure sexual satisfaction from receptive anal intercourse to help care for men engaging in receptive anal intercourse after PCa treatment.
\end{abstract}

Keywords: gay men, health-related quality of life, prostate cancer, sexual function

\section{Introduction}

A N ESTIMATED 3.1 MILLION men live with a prostate cancer (PCa) diagnosis in the United States. ${ }^{1}$ In 2020, an estimated 191,930 new cases will be diagnosed. ${ }^{2}$ In the United States, $1.5 \%-6 \%$ of the general adult population identifies as a man who has sex with men (MSM). ${ }^{3}$ By extrapolation, there are between 46,500 and 186,000 MSM in the United States living with a PCa diagnosis. Despite a sizeable population of MSM with $\mathrm{PCa}, \mathrm{PCa}$ in this population has been understudied until recently, with much of the literature based on qualitative data. ${ }^{4}$ This is largely because data on sexual orientation and sexual practices are not collected routinely with large-scale cancer surveillance surveys. ${ }^{5}$

Management of localized PCa typically involves active surveillance, surgery, or radiation therapy. ${ }^{6}$ Although overall survival for men treated for localized PCa is favorable, the side effects of treatment can have a profound impact on quality of life. ${ }^{7}$ Sexual side effects with treatment can be particularly bothersome, and include erectile, ejaculatory, and orgasmic dysfunction, penile shortening, and Peyronie's disease. ${ }^{8}$ To date, much of the literature on the impact of PCa on sexual function has focused on heterosexual men. ${ }^{7,9}$ Moreover, instruments commonly used to assess sexual function were developed in cohorts where the sexual orientation of the participants was unknown, or validated in predominantly heterosexual cohorts. For instance, in the development of the Expanded Prostate Cancer Index Composite (EPIC) sexual function assessment, no data on the sexual orientation of the cohort are provided. ${ }^{10}$ The Male Sexual Health Questionnaire ejaculation scale did include gay and bisexual men in its development, but they accounted for only 179 out of the

Department of Urology, Feinberg School of Medicine, Northwestern University, Chicago, Illinois, USA. 
8000 participants. ${ }^{10,11}$ As a result, tools measuring sexual function in men focus on erectile function. For gay men who engage in receptive anal intercourse, sexual pleasure may be derived nongenitally, and existing sexual function questionnaires may not apply to them, or may measure sexual function incompletely.

The EPIC sexual function assessment is an expansion of the UCLA-Prostate Cancer Index, capturing additional concerns pertaining to radiation, radical prostatectomy, and androgen deprivation when assessing function and bother. ${ }^{10,12}$ The objective of this study was to determine if there are sexual function domains relevant to gay men that are not captured by the EPIC sexual function assessment.

\section{Methods}

\section{Study cohort and data collection}

Owing to the anonymous collection of data through online surveys, we received an institutional review board (IRB) exemption from Northwestern University's IRB. Informed consent was obtained online from all participants before commencing the survey. Convenience sampling was used to recruit the participants. Gay men were predominantly recruited from Prime Timers, a social organization for older gay and bisexual men, as well as from flyers posted in bars and clinics. Participants were also recruited through researchmatch.org, an online recruitment registry used to facilitate the recruitment of volunteers for research studies. ${ }^{13,14}$

Men with a diagnosis of PCa identifying as gay, $>50$ years, residing in the United States, and with the ability to communicate in English were eligible. An e-mail with an invitation to participate and a link to the survey was sent to eligible men. Participants were not compensated for completing the surveys, and participation was voluntary. Given the multiple methods used to recruit participants, it was not possible to calculate a survey response rate. In all, 53 gay men with a diagnosis of PCa met the inclusion criteria for the study. Men were categorized as gay if they identified as gay and responded that they are attracted to or had sex with men and were not attracted to women. Results were collected and stored in REDCap (Vanderbilt University, Nashville, TN). ${ }^{15}$

\section{Survey development and measures}

We constructed an anonymous survey to assess for differences regarding the applicability of the EPIC sexual function assessment tool in gay men to identify areas of sexual function that are not measured by existing sexual function questionnaires. We collected data on age, race/ethnicity, educational attainment, income level, and relationship status as well as sexual activity. Sexual activity was determined by asking participants if they had engaged in vaginal, anal, or oral sex with a partner or masturbated alone in the preceding month and year. Participants were also asked about the number of sexual partners within those time frames. Separately, participants were asked if they considered themselves to be in a monogamous relationship if they indicated that they were in a relationship.

Participants were asked whether they believed the prostate was a source of sexual pleasure and whether it was important to measure sexual satisfaction as it relates to receptive anal intercourse when assessing sexual function after treatment for PCa. Responses to the first question were recorded as, "yes, no, or unsure" in multiple choice format, and responses to the second question were recorded on a Likert-type scale, with the following responses: "not at all important, important, neutral, important, and very important." In analyzing the question asking participants if it was important to measure sexual satisfaction as it relates to receptive anal intercourse after PCa treatment, we combined "not at all important" and "not important" (categorized as "no") and "important" and "very important" (categorized as "yes"). We presented the EPIC sexual function tool to participants and examined whether they felt it was applicable to their sexual practices, with five possible responses ranging from "very applicable" to "not at all applicable." Participants were also given the ability to comment (using free text) on why the tool was not applicable. These comments were recorded and analyzed by two reviewers and grouped into common themes based on the content of the responses.

\section{Statistical analysis}

Descriptive statistics were generated for general demographic information as well as for responses to questions about sexual activity in the preceding month and year, rates of monogamy, and number of sexual partners in the preceding month and year for the overall cohort. Similarly, descriptive statistics were generated with regard to the applicability of the EPIC sexual function assessment as it relates to measuring erectile function. How participants perceived the importance of measuring sexual satisfaction as it relates to receptive anal intercourse, and their views on the prostate as a source of sexual pleasure were also measured, and analyzed using a chi-squared test. Because we hypothesized that the answers to these questions may differ in men who engaged in receptive anal intercourse and those who did not, we stratified analyses by engagement in receptive anal intercourse (yes/no) within the previous year, and a chi-squared test was used to assess for differences in responses between these groups, across all categories.

\section{Results}

Fifty-three gay men with PCa responded to the survey. Men who identified as bisexual or asexual, and men who did not disclose their sexual orientation were excluded from the analysis. Bisexual men were excluded from our analysis as there is evidence that gay and bisexual men have different experiences with cancer survivorship. ${ }^{16}$ The mean age of the cohort was 74.9 years (standard deviation 7.8 ). The majority $(98.1 \%)$ of respondents were White/non-Hispanic. More than half $(56.6 \%)$ of the cohort had a graduate degree, and a combined $54.7 \%$ of participants identified as being single, or being divorced, separated, or widowed. (Table 1).

\section{Sexual activity and monogamy}

The cohort reported on sexual activity in the preceding month and year. These results are shown in Table 2. In summary, $71.7 \%$ of participants had sex with a partner in the preceding year. Equal proportions of men reported engaging in receptive anal intercourse and insertive anal intercourse in the preceding year (19.2\% for both). The most common form of sexual activity reported was solitary masturbation (75.0\% of men in the preceding year), and the most common 
Table 1. Participant Characteristics

Total, $n$
Age in years, mean (SD)
Race/ethnicity, $n(\%)$
White/non-Hispanic
Non-White/Hispanic
Annual income, $n(\%)$
$\$ 0-\$ 49,999$
$\$ 50,000-\$ 99,999$
$\$ 100,000+$
Education, $n(\%)$
HS/GED/trade/some college
Undergraduate degree
Graduate degree
Relationship status, $n(\%)$
Married/civil union
Has partner
Divorced/separated/widowed
Single

All questions were voluntary; therefore, columns do not add up to 53 if participants declined to answer a question. All percentages calculated as a fraction of the total sample size $(n=53)$.

GED, general educational development; HS, high school; SD, standard deviation.

sexual activities performed with a partner were giving and receiving oral sex (59.6 and $57.7 \%$, respectively). Of the 19 men who reported being in a relationship, 15 of them reported being monogamous $(78.9 \%)$, and $37.7 \%$ of all respondents reported having more than one sexual partner in the preceding year (Table 3).

\section{Applicability of the EPIC sexual function tool}

A majority of gay men $(39 / 51,76.5 \%)$ found the EPICSexual Function domain (EPIC-SF) to be applicable to them when measuring erectile function. In analyzing the free-text responses, respondents noted that the questionnaire lacked

Table 2. Sexual Activity in the Preceding MONTH AND YEAR

\begin{tabular}{lcr}
\hline & $\mathrm{n}$ & $\%$ \\
\hline In the preceding month & & \\
Sex with a partner & $24 / 53$ & 45.3 \\
Vaginal intercourse & $1 / 53$ & 1.9 \\
Insertive anal intercourse & $5 / 52$ & 9.6 \\
Receptive anal intercourse & $6 / 52$ & 11.5 \\
Gave oral sex & $19 / 50$ & 38.0 \\
Received oral sex & $18 / 49$ & 36.7 \\
Solitary masturbation & $39 / 52$ & 75.0 \\
In the preceding year & & \\
Sex with a partner & $38 / 53$ & 71.7 \\
Vaginal intercourse & $1 / 51$ & 2.0 \\
Insertive anal intercourse & $10 / 52$ & 19.2 \\
Receptive anal intercourse & $10 / 52$ & 19.2 \\
Gave oral sex & $31 / 52$ & 59.6 \\
Received oral sex & $30 / 52$ & 57.7 \\
Solitary masturbation & $39 / 52$ & 75.0 \\
\hline Although the total number of participants was 53, each participant \\
was allowed to select multiple answer choices. The denominator is \\
provided for each question.
\end{tabular}

Table 3. Rates of Monogamy and Number of Sexual Partners in the Preceding MONTH AND YEAR

\begin{tabular}{lcc}
\hline & $\mathrm{n}$ & $\%$ \\
\hline Monogamous $^{\mathrm{a}}$ & 15 & 78.9 \\
Sexual partners in the preceding month $(n=51)$ & \\
0 & 24 & 47.1 \\
1 & 18 & 35.3 \\
$>1$ & 9 & 17.7 \\
Sexual partners in the preceding year $(n=53)$ & \\
0 & 16 & 30.2 \\
1 & 17 & 32.1 \\
$>1$ & 20 & 37.7 \\
\hline
\end{tabular}

${ }^{a}$ Fifteen out of 19 men who reported being in a relationship reported being monogamous. The percentage reported accounts for men in a relationship only.

specific questions about masturbatory function, as well as quality of orgasm, which were thought to be important aspects of sexual function. Respondents were also dissatisfied with the lack of questions about receptive anal intercourse and felt that this limited the scope of the questionnaire.

\section{The prostate as a source of sexual pleasure}

Table 4 shows that a majority of respondents felt that the prostate is a source of sexual pleasure (64.2\%), compared with $13.2 \%$ who did not think so, and $22.6 \%$ who were unsure $(p<0.0001)$. More than half of the men felt it important to measure sexual satisfaction related to receptive anal intercourse $(52.8 \%)$. Significantly more men who engaged in receptive anal intercourse in the preceding year felt the prostate was a source of sexual pleasure compared with men who did not engage in this activity ( $100 \%$ vs. $57.1 \%, p=0.048)$. No men engaging in receptive anal intercourse felt unsure about the prostate as a source of pleasure, compared with $26.2 \%$ of men who did not engage in this activity. Ninety percent of men who engaged in receptive anal intercourse versus $45.2 \%$ of men who did not engage in receptive anal intercourse felt it important to measure sexual satisfaction as it relates to receptive anal intercourse after PCa treatment (Table 5).

Table 4. Views on the Prostate as a Source of Sexual Pleasure and the Importance of Measuring Sexual Satisfaction Related to Receptive Anal Intercourse After Treatment For Prostate Cancer

\begin{tabular}{|c|c|c|c|}
\hline & $\mathrm{n}$ & $\%$ & $\mathrm{p}$ \\
\hline \multicolumn{4}{|c|}{ The prostate as source of sexual pleasure } \\
\hline Yes & 34 & 64.2 & \multirow[t]{3}{*}{$<0.0001$} \\
\hline No & 7 & 13.2 & \\
\hline Unsure & 12 & 22.6 & \\
\hline \multicolumn{4}{|c|}{$\begin{array}{l}\text { Important to measure sexual satisfaction with RAI after PCa } \\
\text { treatment }\end{array}$} \\
\hline Yes & 28 & 52.8 & \multirow[t]{3}{*}{0.01} \\
\hline Neutral & 17 & 32.1 & \\
\hline No & 8 & 15.1 & \\
\hline
\end{tabular}

$p$ values apply to comparisons across all three categories for both questions.

$\mathrm{PCa}$, prostate cancer; RAI, receptive anal intercourse. 
Table 5. Views on the Prostate as a Source of Sexual Pleasure and the Importance of Measuring Sexual Satisfaction Related to Receptive Anal Intercourse After Treatment for Prostate Cancer, Stratified by Engagement IN RECEPTIVE ANAL INTERCOURSE IN THE PRECEDING YeAR

\begin{tabular}{|c|c|c|c|c|c|}
\hline & \multicolumn{2}{|c|}{$\begin{array}{l}\text { Engaged in } \\
\text { receptive anal } \\
\text { intercourse }\end{array}$} & \multicolumn{2}{|c|}{$\begin{array}{l}\text { Not engaged in } \\
\text { receptive anal } \\
\text { intercourse }\end{array}$} & \multirow[b]{2}{*}{$\mathrm{p}$} \\
\hline & $\mathrm{n}$ & $\%$ & $\mathrm{n}$ & $\%$ & \\
\hline \multicolumn{6}{|c|}{ Prostate as source of sexual pleasure } \\
\hline Yes & 10 & 100.0 & 24 & 57.1 & 0.048 \\
\hline No & 0 & 0.0 & 7 & 16.7 & \\
\hline Unsure & 0 & 0.0 & 11 & 26.2 & \\
\hline \multicolumn{6}{|c|}{$\begin{array}{l}\text { Important to measure sexual satisfaction as it relates } \\
\text { to receptive anal intercourse after PCa treatment }\end{array}$} \\
\hline Yes & 9 & 90.0 & 19 & 45.2 & 0.059 \\
\hline Neutral & 1 & 10.0 & 16 & 38.1 & \\
\hline No & 0 & 0.0 & 7 & 16.7 & \\
\hline
\end{tabular}

$p$ values apply to comparisons across all three categories for both questions.

\section{Discussion}

To our knowledge, we report findings from the first quantitative study on the utility and applicability of a routinely used sexual function assessment in gay men. Overall, our study demonstrates that sexual function in gay menparticularly as it relates to receptive anal intercourse-is measured incompletely when assessing sexual function after PCa treatment.

In our study, the overall reported level of sexual activity with a partner in the preceding year was higher than levels reported by Lindau et al. in a sample of 547 community dwelling adult men between 65 and 74 years. ${ }^{17}$ These findings are consistent with a prior study showing greater levels of sexual activity in gay men compared with heterosexual men. ${ }^{18}$ We did, however, note high levels of monogamy within men who reported being in a relationship (78.9\%). This finding is in contrast with previously published data showing lower rates of monogamy in gay men. ${ }^{18}$ This may be due to differences in behavior among older gay men who have undergone treatment for PCa compared with gay men without a PCa diagnosis, or may be due to sampling error given the small sample size of our cohort. Further study is needed with a larger cohort to validate this finding.

There is evidence that gay and bisexual men experience a decline in health-related quality of life after PCa treatment, ${ }^{19,20}$ with increased rates of psychological distress from erectile dysfunction, ${ }^{21}$ which may relate to expectations of having an active sexual life that continues into their later years. ${ }^{21}$ Moreover, sexual factors often play an important role in the identities of gay men, ${ }^{22,23}$ and a loss of sexual function can be particularly devastating to gay men who "came out" later in life, as they miss the opportunity to explore their recently accepted sexuality. ${ }^{24}$ Men who are nonmonogamous have the additional challenge of navigating new sexual encounters, and changes in erectile and rectal function can pose challenges to men engaging in casual sex when negotiating roles in sex with unfamiliar partners. ${ }^{23}$
Taken together, this highlights the importance of thorough preoperative counseling regarding the sexual side effects of treatment with gay men, and discussing all the different management options and their attendant risks, to allow these patients to weigh the risks and benefits as they apply to this patient population, in making a clinical decision.

We asked respondents to rate the applicability of a wellknown validated instrument (the EPIC sexual function assessment), which is representative of commonly used sexual assessments in the clinical setting, ${ }^{10}$ and the majority of participants in our study found this tool to be applicable to them as a measure of erectile function. However, as sexual function in gay men may encompass erectile function as well as functioning related to receptive anal intercourse, a followup question regarding whether the prostate serves as a source of sexual pleasure was posed. A significant number of men $(64.2 \%)$ felt the prostate to be a source of sexual pleasure, and $52.8 \%$ felt it important to measure sexual satisfaction with receptive anal intercourse after treatment for PCa. All gay men engaging in receptive anal intercourse viewed the prostate as a source of sexual pleasure compared with $57.1 \%$ of men not engaging in receptive anal intercourse. More men engaging in receptive anal intercourse felt it important to measure sexual satisfaction related to receptive anal intercourse after treatment for PCa, compared with men not engaging in this activity. However, our analyses did not reach statistical significance on this last measure, which is likely due to inadequate power to show a difference, given the small sample size.

Our study calls to attention the need for a validated instrument measuring sexual satisfaction related to anal intercourse, particularly (although not exclusively) in gay men engaging in receptive anal intercourse. This need was noted in a prior pilot study of $15 \mathrm{MSM}$ treated for $\mathrm{PCa},{ }^{25}$ and our findings are congruent with this smaller study. There have been previous attempts to make sexual function questionnaires more applicable to MSM. For instance, Coyne et al. modified the International Index of Erectile Function and tailored it to MSM to include erectile function during oral and anal sex (receptive and insertive) but did not specifically measure sexual pleasure from receptive anal intercourse. ${ }^{26}$

Having an instrument that measures pleasure with receptive anal intercourse, that is separate from existing questionnaires assessing erectile function, could serve many purposes. Such an instrument could help to determine how different treatments for $\mathrm{PCa}$ affect function related to receptive anal intercourse, which could assist men engaging in receptive anal intercourse in making an informed decision about treatment choice. In addition, offering such an instrument could help physicians engage with gay men who practice receptive anal intercourse, help gay men feel cared for after treatment, and help chart the trajectory of their sexual recovery as it pertains to receptive anal intercourse after PCa treatment. Indeed, questionnaires that do not account for sexual behaviors more commonly practiced by nonheterosexual men can be perceived as heterosexist and cause frustration among gay men seeking postoperative care, ${ }^{27}$ which may lead to alienation and poorer outcomes in these men.

\section{Limitations}

Given the varied methods of recruitment, we were unable to calculate a response rate, and our sample may be subject to 
response bias. Our cohort represents a highly educated population (56.6\% of participants reported some level of postgraduate education). We also recruited heavily from a social organization for gay men, as well as researchmatch.org. Whether the trends seen in our study are generalizable to men with lower levels of education, and those outside of our recruitment populations warrants further investigation. In addition, the majority of participants in this study were Caucasian, and all of the men identified as gay, and the results of this study may not be generalizable to racial minority men and bisexual men.

It should be emphasized that our study is a hypothesisgenerating pilot study. Further study looking at a larger cohort of men with a PCa diagnosis is needed, with attention paid to the type of treatment received (commonly, surgical or radiation therapy) as well as the time since diagnosis, as a patient's experience of survivorship can alter with time. Furthermore, a limitation of our current study is that participants were not queried about bothersome bowel symptoms post-treatment. For men who engage in receptive anal intercourse and experience bowel symptoms post-treatment, it is likely that sexual satisfaction and bowel bother are related. Further exploration of the intersection of these two areas of quality of life outcomes is needed in future studies.

From an anatomic standpoint, it is likely that a combination of nerves is responsible for pleasure with receptive anal intercourse, including the pudendal (anus), pelvic (rectum), and hypogastric (prostate) nerves, ${ }^{28}$ and pleasure from receptive anal intercourse is likely not solely derived from the prostate (the focus of our questions). However, this does not change the need for a validated instrument for measuring satisfaction associated with receptive anal intercourse, which would account for pleasure regardless of the specific physical components contributing to it. Finally, our survey asked participants specifically about sexual satisfaction as it relates to receptive anal intercourse, which may have excluded men who stimulate their prostate in ways other than receptive anal intercourse. Further study is needed on how these men view the applicability of current surveys of sexual function.

\section{Conclusion}

There are currently no validated instruments measuring sexual function related to receptive anal intercourse. Creating such an instrument that is separate from tools measuring erectile function can help us understand how different treatments for $\mathrm{PCa}$ affect receptive anal intercourse, and help patients choose treatments based on side-effect profiles. It would also help to assess recovery in patients engaging in receptive anal intercourse after PCa treatment and help improve cancer survivorship in this population.

\section{Author Disclosure Statement}

No competing financial interests exist.

\section{Funding Information}

No funding was received for this article.

\section{References}

1. National Cancer Institute: Cancer Stat Facts: Prostate Cancer. 2018. Available at https://seer.cancer.gov/ statfacts/html/prost.html Accessed May 3, 2019.

2. American Cancer Society: Key Statistics for Prostate Cancer. 2020. Available at www.cancer.org/cancer/ prostate-cancer/about/key-statistics.html Accessed April 26, 2020.

3. Grey JA, Bernstein KT, Sullivan PS, et al.: Estimating the population sizes of men who have sex with men in US states and counties using data from the American Community Survey. JMIR Public Health Surveill 2016;2:e14.

4. Simon Rosser BR, Merengwa M, Capistrant BD, et al.: Prostate cancer in gay, bisexual, and other men who have sex with men: A review. LGBT Health 2016;3:32-41.

5. Boehmer U, Miao X, Ozonoff A: Cancer survivorship and sexual orientation. Cancer 2011;117:3796-3804.

6. Gray PJ, Lin CC, Cooperberg MR, et al.: Temporal trends and the impact of race, insurance, and socioeconomic status in the management of localized prostate cancer. Eur Urol 2017;71:729-737.

7. Sanda MG, Dunn RL, Michalski J, et al.: Quality of life and satisfaction with outcome among prostate-cancer survivors. N Engl J Med 2008;358:1250-1261.

8. Benson CR, Serefoglu EC, Hellstrom WJ: Sexual dysfunction following radical prostatectomy. J Androl 2012;33: 1143-1154.

9. Resnick MJ, Koyama T, Fan KH, et al.: Long-term functional outcomes after treatment for localized prostate cancer. N Engl J Med 2013;368:436-445.

10. Wei JT, Dunn RL, Litwin MS, et al.: Development and validation of the expanded prostate cancer index composite (EPIC) for comprehensive assessment of health-related quality of life in men with prostate cancer. Urology 2000; 56:899-905.

11. Rosen RC, Catania JA, Althof SE, et al.: Development and validation of four-item version of Male Sexual Health Questionnaire to assess ejaculatory dysfunction. Urology 2007;69:805-809.

12. Vertosick EA, Vickers AJ, Cowan JE, et al.: Interpreting patient reported urinary and sexual function outcomes across multiple validated instruments. J Urol 2017;198: 671-677.

13. Harris PA, Scott KW, Lebo L, et al.: ResearchMatch: A national registry to recruit volunteers for clinical research. Acad Med 2012;87:66-73.

14. Tan MH, Thomas M, MacEachern MP: Using registries to recruit subjects for clinical trials. Contemp Clin Trials 2015;41:31-38.

15. Harris PA, Taylor R, Thielke R, et al.: Research electronic data capture (REDCap) — a metadata-driven methodology and workflow process for providing translational research informatics support. J Biomed Inform 2009;42: 377-381.

16. Rust PC: Bisexuality: The state of the union. Annu Rev Sex Res 2002;13:180-240.

17. Lindau ST, Schumm LP, Laumann EO, et al.: A study of sexuality and health among older adults in the United States. N Engl J Med 2007;357:762-774.

18. Gotta G, Green RJ, Rothblum E, et al.: Heterosexual, lesbian, and gay male relationships: A comparison of couples in 1975 and 2000. Fam Process 2011;50:353-376.

19. Torbit LA, Albiani JJ, Crangle CJ, et al.: Fear of recurrence: The importance of self-efficacy and satisfaction with care in 
gay men with prostate cancer. Psychooncology 2015;24: 691-698.

20. Ussher JM, Perz J, Kellett A, et al.: Health-related quality of life, psychological distress, and sexual changes following prostate cancer: A comparison of gay and bisexual men with heterosexual men. J Sex Med 2016;13:425-434.

21. Ussher JM, Perz J, Rose D, et al.: Threat of sexual disqualification: The consequences of erectile dysfunction and other sexual changes for gay and bisexual men with prostate cancer. Arch Sex Behav 2017;46:2043-2057.

22. Fergus KD, Gray RE, Fitch MI: Sexual dysfunction and the preservation of manhood: Experiences of men with prostate cancer. J Health Psychol 2002;7:303-316.

23. Lee TK, Handy AB, Kwan W, et al.: Impact of prostate cancer treatment on the sexual quality of life for men-whohave-sex-with-men. J Sex Med 2015;12:2378-2386.

24. Ussher JM, Rose D, Perz J: Mastery, isolation, or acceptance: Gay and bisexual men's construction of aging in the context of sexual embodiment after prostate cancer. J Sex Res 2017;54:802-812.

25. Lee TK, Breau RH, Eapen L: Pilot study on quality of life and sexual function in men-who-have-sex-with-men treated for prostate cancer. J Sex Med 2013;10:2094-2100.
26. Coyne K, Mandalia S, McCullough S, et al.: The International Index of Erectile Function: Development of an adapted tool for use in HIV-positive men who have sex with men. J Sex Med 2010;7:769-774.

27. Hoyt MA, Frost DM, Cohn E, et al.: Gay men's experiences with prostate cancer: Implications for future research. J Health Psychol 2020;25:298-310.

28. Komisaruk BR, Whipple B, Nasserzadeh S, Beyer-Flores C: Orgasms and relationships. In: The Orgasm Answer Guide. Baltimore, MD: The Johns Hopkins University Press, 2010, pp 108-109.

Address correspondence to: Channa Amarasekera, MD Department of Urology Feinberg School of Medicine Northwestern University 676 N. St. Clair, Arkes 23-010 Chicago, IL 60611

USA

E-mail: channa.amarasekera@northwestern.edu 